\title{
Endurecimiento superficial de aceros y fundiciones mediante láser de $\mathrm{Nd}$ :
} YAG(*)

\author{
R. Sagaró, H. Carvajal ${ }^{*}$ J.S. Ceballos*, R. Cabrera* y J. Mascarell ${ }^{*}$
}

Resumen En el trabajo se presentan los resultados del tratamiento térmico superficial, con técnica láser, de superficies metálicas. Con el empleo de un láser de $\mathrm{Nd}$ :YAG se procedió al endurecimiento superficial de los aceros AISI 1045, W1, W112 y fundiciones de tipo laminar y nodular. Se presentan los resultados del tratamiento térmico superficial atendiendo al parámetro velocidad de barrido del haz de láser (V). De igual forma se estudian las microestructuras metalográficas obtenidas. En todos los casos, se obtienen valores de microdureza Vickers que oscilan entre los 500-900 HV (incrementos de trescuatro veces respecto al material base). Los resultados obtenidos permiten afirmar que, en todos los casos estudiados, el incremento de la velocidad de barrido provocó una disminución de la profundidad de la capa endurecida, en correspondencia con otros resultados expuestos en la literatura especializada. Las experiencias del tratamiento superfical con láser en las muestras ensayadas muestran una tendencia a la disminución de la profundidad del endurecimiento con el incremento del contenido de carbono. Como complemento, se realizaron ensayos tribológicos que demostraron un incremento en la resistencia al desgaste, comparativamente con tratamientos convencionales y con fundiciones patrones (no tratadas). El comportamiento del coeficiente de fricción mostró una tendencia generalizada a la disminución en las muestras con tratamiento térmico superficial con láser.

Palabras clave Endurecimiento superficial por láser. Tratamiento térmico por láser. Modificación superficial. Comportamiento tribológico.

\section{Nd:Yag laser surface hardening for steels and cast iron}

Abstract

Keywords
This work relates with heat treatment by laser of metallic surfaces. The hardening of steels AISI 1045, W1, W112 and flake and nodular cast irons was carried out using a Nd: YAG laser. Operation parameters of laser like beam power $(\mathrm{P})$ and traverse speed of the laser Spot (V) are closely related to the depth of hardening. The authors present some analyses on metallographic studies. In all cases Vickers microhardness valves of about 500-900 HV (three or four times higher than original substrates). Relations between microhardness and depth of hardening are presented for the processed materials. Experimental results showed that the scan speed increase produces a decrease on depth of hardening as reported by others researchers. On the other hand, the samples with higher carbon content show lower hardenining depth. In addition, tribological tests were carried out. These tests show wear resistance when compared with conventional heat treatment. The frictional coefficient decrease in the samples with laser surface treatment.

Laser surface hardening. Laser heat treatment. Surface modification. Tribological behaviour.

(•) Trabajo recibido el día 25 de Mayo de 2001 y aceptado en su forma final el día 22 de Mayo de 2001

(*) Facultad de Ingeniería Mecánica, Universidad de Oriente, Cuba.

$\left.{ }^{(* *}\right)$ Escuela Superior Politécnica de Chimborazo, Ecuador. 


\section{INTRODUCCIÓN}

El incremento de las aplicaciones tecnológicas, en el campo metalúrgico, del láser y el creciente interés que ha desatado en determinados procesos industriales, vienen dados por sus características intrínsecas, tales como: la posibilidad de la absorción superficial de la energía luminosa, el control exacto de las zonas sobre las que se desea trabajar, el control de la potencia del láser que permite diferentes tratamientos, un proceso fácilmente automatizable y una fuente de energía totalmente limpia ${ }^{[1]}$.

El tratamiento térmico superficial de metales con láser persigue obtener un aumento de dureza en la superficie, calentando la capa superficial del acero hasta la temperatura de austenización, con un haz láser enfocado sobre el material, lográndose el enfriamiento por conducción al resto del material de la pieza, sin necesidad de agentes externos. Como todo temple superficial, tiene como objetivo obtener una elevada dureza en la capa superficial sin alterar las propiedades del substrato. Parámetros como potencia incidente del láser, diámetro del haz, absorción superficial de energía, velocidad de barrido, propiedades termofísicas del substrato, así como su composición química, determinan la profundidad endurecida y su dureza ${ }^{[2-4]}$.

El estudio realizado presenta la idoneidad del proceso de temple superficial por láser, prestando especial interés a la velocidad de barrido del haz como uno de los parámetros de operación del láser, que regulan el proceso, sobre cinco materiales férreos diferentes. De igual forma, se consideran los resultados del tratamiento láser sobre las características de fricción y desgaste de los materiales ensayados.

\section{PROCEDIMIENTO EXPERIMENTAL}

Para la realización de los experimentos se utilizó una instalación láser del tipo $\mathrm{Nd}$ :YAG de baja potencia acoplado a una mesa X-Y. El haz del láser incide perpendicularmente sobre la muestra mediante la óptica de enfoque y podrá regularse la densidad de potencia requerida variando el diámetro de incidencia del láser, por medio del desenfoque de éste (Fig.1). La tabla I recoge las especificaciones técnicas del láser empleado.

Las mediciones de microdureza Vickers se efectuaron en el microdurómetro SHIMADZU, tipo $\mathrm{N}$. El ensayo se realizó según el procedimiento de la norma ASTM-E384, para una carga aplicada de 50 gr.

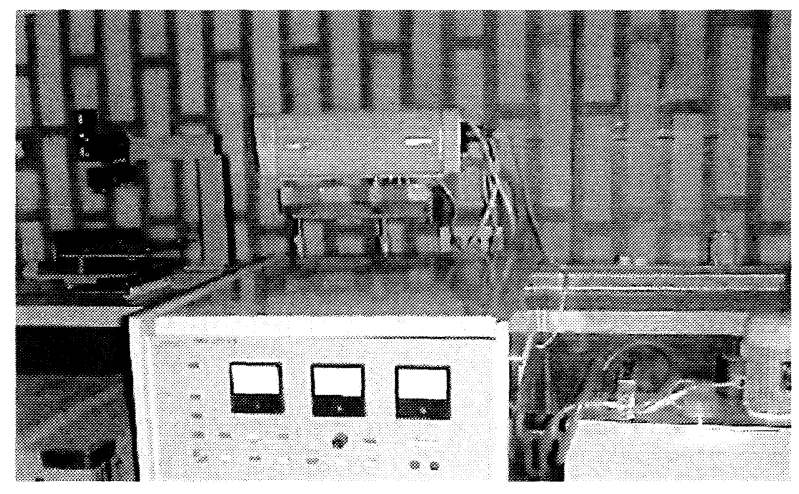

Figura 1. Instalación experimental.

Figure 1. Experimental device.

Tabla I. Parámetros del equipo láser LTI 702

Table I. Parameters of laser device LTI 702

\begin{tabular}{lr} 
Longitud de onda & $1.060 \mathrm{~nm}$ \\
Spót & $3 \mathrm{~mm}$ \\
Divergencia & $2 \times 10^{-3} \mathrm{rad}$. \\
Potencia de salida garantizada & $120 \mathrm{~W}$ \\
Régimen de trabajo & continuo \\
Modo & TEM $_{01}$ \\
\hline
\end{tabular}

Los ensayos se realizaron en cinco materiales férreos: aceros AISI 1045, W1 y W112, así como fundiciones grises laminares y nodulares. La tabla II recoge la composición química de los materiales empleados.

Se emplearon probetas prismáticas de dimensiones $40 \times 10 \times 5 \mathrm{~mm}$. El tratamiento con láser, de las muestras, se realizó sin superposición de pasadas del haz de láser. Para garantizar la mayor absorción en las superficies metálicas se aplicó un recubrimiento de pintura negra mate en una capa de 0,01 $\mathrm{mm}$. Para cada uno de los materiales ensayados se utilizaron tres probetas, sobre las que se realizaron tres pasadas.

Para la realización de estudios metalográficos se procedió al corte transversal de las muestras. El corte de las probetas se efectuó por su sección central, utilizando un disco abrasivo y refrigeración adecuada, de forma tal que el calentamiento fuera mínimo y evitar así transformaciones estructurales que pudieran afectar los resultados. La medida de los espesores afectados por el tratamiento y los valores de microdureza se obtuvieron siempre, en el centro de la región afectada.

Los materiales se trataron mediante la instalación láser esquematizada en la figura 1. Como 
Tabla II. Composición química de los materiales ensayados

Table II. Chemical composition of tested materials

\begin{tabular}{|c|c|c|c|}
\hline Denominación & Composición & Características & Dureza (HV) \\
\hline Acero AISI 1045 & $0,45 \% \mathrm{C}, 0,7 \% \mathrm{Mn}, 0,3 \% \mathrm{Si},<0,03 \% \mathrm{Cr}$ & Perlita y ferrita en los límites del grano & 210 \\
\hline Acero AISI W1 & $0,75-0,84 \% \mathrm{C}, 0,15-0,4 \% \mathrm{Mn},<0,15 \% \mathrm{Cr}$ & $\begin{array}{l}\text { Estructura perlítica con partículas } \\
\text { esferoidales de cementita }\end{array}$ & 217 \\
\hline Acero AISI W112 & $1,25-1,35 \% \mathrm{C}, 0,15-0,35 \% \mathrm{Mn},<0,15 \% \mathrm{Cr}$ & $\begin{array}{l}\text { Estructura perlítica con mayor cantidad } \\
\text { de partículas esferoidales de cementita }\end{array}$ & 224 \\
\hline Fundición gris laminar & $3,4 \% \mathrm{C}, 4 \% \mathrm{CE}, 0,59 \% \mathrm{Mn}, 18 \% \mathrm{Si}, 0,18 \% \mathrm{Cr}$ & Láminas de grafito en una matriz perlítica & 234 \\
\hline Fundición gris nodular & $3,5 \% \mathrm{C}, 4,3 \% \mathrm{CE}, 0,3 \% \mathrm{Mn}, 2,4 \% \mathrm{Si}$ & $\begin{array}{l}\text { Nódulos de grafito rodeados de ferrita } \\
\text { libre en una matriz perlítica }\end{array}$ & 297 \\
\hline
\end{tabular}

parámetro a considerar se definió la profundidad del endurecimiento, como la máxima profundidad a la que se observó transformación estructural en cada una de las pasadas del haz de láser. Los parámetros del láser que se utilizaron se indican en la tabla III.

Se realizaron ensayos de desgaste comparando los resultados del tratamiento con láser de los aceros estudiados, con tratamientos térmicos convencionales y las fundiciones originales sin tratamiento láser. Los patrones comparativos fueron:

- Acero AISI 1045, con temple a $820-850{ }^{\circ} \mathrm{C}$, con enfriamiento en agua y sin revenido, $\mathrm{HV}=700$.

- Acero AISI W1, temple por alta frecuencia, $\mathrm{HV}=926$.

- Acero AISI W112, temple en sales HV=941.

- Las fundiciones en su estado de partida (Tabla II).

Los ensayos tribológicos se realizaron en un tribómetro del tipo bloque-cilindro, bajo condiciones de fricción seca. Las condiciones experimentales fueron: velocidad de deslizamiento $\mathrm{V}=0,42 \mathrm{~m} / \mathrm{s}$, bajo carga normal $\mathrm{Fn}=100 \mathrm{~N}$. La duración del ensayo fue de una hora.

En calidad de contracuerpo metálico se emplearon cilindros de acero $65 \mathrm{Mn} 4$ de dimensión $\phi 40$ $\times 12 \mathrm{~mm}, \mathrm{Ra}=6,5 \mu \mathrm{m}$ y $\mathrm{HV}=548-593$. Las probe-

Tabla III. Parámetros del láser

Table III. Laser parameters

\begin{tabular}{ccccc}
\hline Potencia, W & Diámetro del haz & \multicolumn{3}{c}{ Velocidades de pasada, $\mathrm{mm} / \mathrm{s}$} \\
\hline 100 & $1 \mathrm{~mm}$ & 3,5 & 4,5 & 5,5 \\
\hline
\end{tabular}

tas planas de cada uno de los materiales ensayados, de dimensión $10 \times 10 \times 12 \mathrm{~mm}$, se trataron previamente con láser, con superposición de pasadas conforme a las condiciones prefijadas de los parámetros operacionales del láser, de manera tal que cubrieran el área de contacto. Los valores de rugosidad posteriores al TTSL fueron de $\mathrm{Ra}=0,8 \mu \mathrm{m}$.

Las medidas de desgaste se realizaron por el método de diferencia en peso, antes y después de cada experimento, en una balanza analítica de 0,0001 gr de precisión. Las medidas del coeficiente de fricción se obtuvieron, para cada instante, a través del registrador gráfico del tribómetro.

\section{RESULTADOS Y DISCUSIONES}

En las figuras 2-4 se muestran de forma gráfica los resultados de las medidas de microdureza Vickers (HV) realizadas a diferentes profundidades para cada una de las pasadas del haz láser, para cada una de las velocidades de desplazamiento del haz usadas en el trabajo.

De forma general, en las figuras $2-4$ se observa que los mayores valores de microdureza en la capa superficial se obtuvieron en los aceros AISI W1 y W112, como era de esperar, producto del alto contenido de carbono y las transformaciones microestructurales que de ello se derivan, independientemente de la velocidad de barrido usada en los ensayos. También existió un perfil de microdureza similar en la mayoría los materiales ensayados.

Como se observa en la figura 2, la profundidad de la capa endurecida en los ensayos realizados para una velocidad de barrido de $3,5 \mathrm{~mm} / \mathrm{s}$ fue aproximadamente de $200 \mu \mathrm{m}$, con excepción del acero AISI W112, el cual alcanzó una profundidad endurecida de $100 \mu \mathrm{m}$, lo que está relacionado con la concentración de carbono. Con el incremento 


\section{Pasada 1}

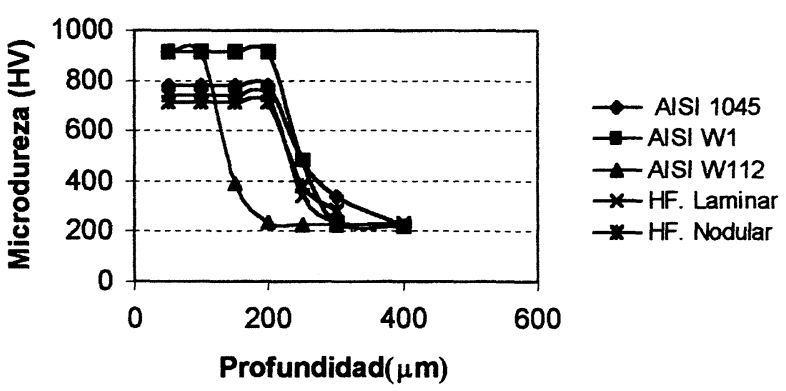

Figura 2. Correlación entre la microdureza (HV) y la profundidad de la capa tratada térmicamente por láser $(v=3,5 \mathrm{~mm} / \mathrm{s})$.

Figure 2. Relation between microhardness (HV) and depth of hardening $(v=3.5 \mathrm{~mm} / \mathrm{s})$.

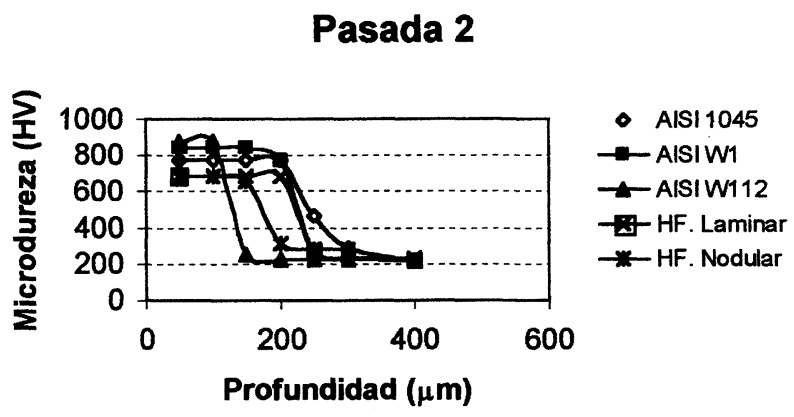

Figura 3. Correlación entre la microdureza (HV) y la profundidad de la capa tratada térmicamente por láser $(\mathrm{v}=4,5 \mathrm{~mm} / \mathrm{s})$.

Figure 3. Relation between microhardness (HV) and depth of hardening $(v=4.5 \mathrm{~mm} / \mathrm{s})$.

\section{Pasada 3}

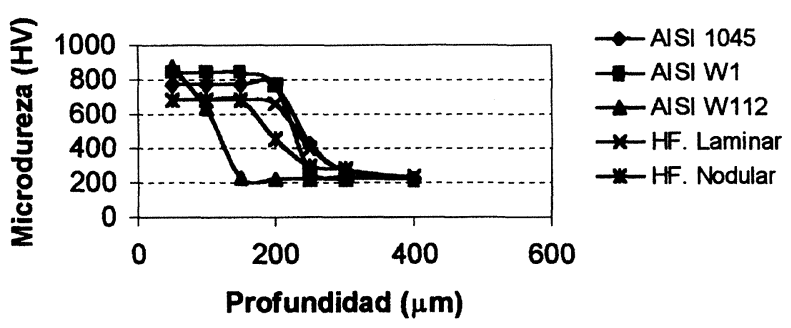

Figura 4. Correlación entre la microdureza (HV) y la profundidad de la capa tratada térmicamente por láser ( $v$ $=5,5 \mathrm{~mm} / \mathrm{s}$ ).

Figure 4. Relation between microhardness and depth of hardening $(v=5.5 \mathrm{~mm} / \mathrm{s})$.

de la velocidad se constató una disminución del espesor de la capa endurecida, como puede observarse en las figuras 3 y 4 .
La variación de la velocidad de barrido en los niveles empleados en los experimentos, si bien provocó variaciones en la profundidad de la capa endurecida, no influyó significativamente en los valores de microdureza de la capa tratada.

La eficacia del tratamiento con láser se demuestra cuando se comparan los resultados del tratamiento de temple convencional (temple 820$850^{\circ} \mathrm{C}$ con enfriamiento en agua y sin revenido) aplicado a un acero AISI 1045, el cual, por este método, alcanza microdurezas del orden de los 700 HV. Sin embargo, la microdureza de la capa endurecida, producto del tratamiento con láser, fue del orden de $780 \mathrm{HV}$, atribuible a la alta razón de enfriamiento asociada con el tratamiento láser y la fina martensita resultante.

Es necesario señalar que el acero AISI 1045, sometido a temple con láser, tenía una microestructura resultante de un normalizado, en el cual la cementita está presente en forma laminar en la microestructura perlítica rodeada de ferrita reticular, todo lo cual dificulta el proceso de austenización. Como se conoce, el normalizado refina el grano y forma ferrita reticular. La gran separación entre las formaciones ferríticas y perlíticas dificultan la austenización completa. De aquí, la importancia de prestar atención a la microestructura de partida $^{[5]}$.

De las figuras anteriores se desprende que las probetas irradiadas con láser presentaron valores de microdureza $\mathrm{HV}$ del orden de 780-916 HV, algo mayores que los alcanzados por un temple convencional $^{[7]}$, resultado muy conveniente por cuanto está soportado por un substrato dúctil que corresponde a la zona no afectada por el láser y que se corresponde con la dureza del material base.

Los resultados apuntan a confirmar lo descrito

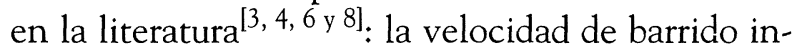
fluye de manera negativa en la penetración del endurecimiento al disminuir el tiempo de interacción y lograr un gradiente térmico insuficiente para austenizar mayores profundidades.

\section{ANÁLISIS DE LA MICROESTRUCTURA}

Las microestructuras observadas pueden agruparse de manera general en tres tipos: la zona no transformada térmicamente, la zona de transición y de total transformación martensítica.

La figura 5 a muestra la microestructura del acero AISI 1045 tratado con láser, en la que se puede observar claramente la zona totalmente transformada y el substrato no transformado. 

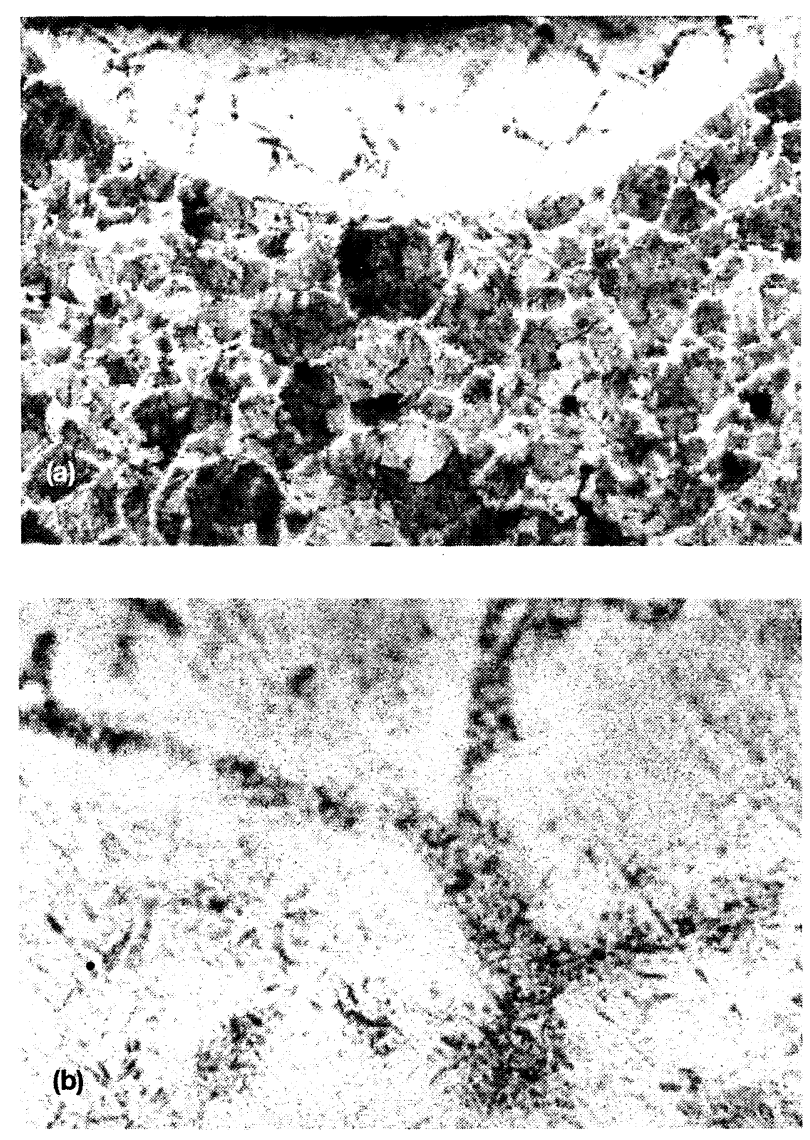

Figura 5. a) Microestructura del acero AISI 1045 (100 X), zona tratada (parte superior blanca) y la no tratada, parte inferior (perlita+ferrita), b) Zona de transición: martensita (blanca) y algo de trostita (gris) (500X).

Figure 5. a) Microstructure of steel AISI 1045 (100X), treated zone (black zone) and untreated below (perlite+ferrite), b) Transition zone: martensite (black) and trostite (grey) (500X).

Se detectó, en todos los casos, en la zona próxima a la superficie, una martensita fina y, en la medida que se avanza en profundidad, una zona de martensita un poco más burda de menor dureza. En la zona de transición se observan zonas parcialmente transformadas (martensita) y zonas sin transformar (perlita y algo de ferrita) y, finalmente, en la zona que corresponde al metal base se mantiene la estructura inicial del substrato de perlita más ferrita.

El análisis del acero AISI W1 mostró que en la zona próxima a la superficie se ha obtenido martensita fina y los carburos casi completamente disueltos, distribuyéndose homogéneamente en la matriz metálica. A mayor profundidad la martensita adoptó una estructura burda de menor dureza y aumentó la cantidad de carburos sin disolver. En la zona de transición aparecieron zonas parcialmente transformadas (martensita más cementita) y zonas sin transformar (perlita más cementita).
La estructura del acero AISI W112, por su parte, presentó después del tratamiento con láser algunas peculiaridades, pues la zona afectada por el calor (ZAC) tenía cuatro zonas bien definidas. La zona no afectada por el láser presentaba una microestructura correspondiente a una perlita globular. Inmediatamente, una zona de transición con perlita globular y trostita y, por encima de ésta, una estructura de martensita fina y perlita globular. Finalmente y correspondiendo con el diámetro del haz una zona exclusiva de martensita muy fina.

Los resultados obtenidos en las fundiciones grises laminares y nodulares, son consecuencia del proceso de solubilización del grafito, como explican otros autores ${ }^{[6 \text { y } 9]}$. En la fundición laminar, por ejemplo, para las diferentes condiciones de realización de los ensayos, los resultados mostraron, en todos los casos, una zona afectada por el calor con una incompleta disolución del grafito en la zona martensítica endurecida por el láser (Fig.6). La incompleta disolución del grafito, en relación con los resultados reportados por Schaaf ${ }^{[9]}$ puede estar relacionada con la baja potencia empleada.

En cuanto a la fundición nodular (Fig. 7), la zona afectada por el láser mostraba una martensita fina con grafito nodular incompletamente disuelto y rodeado por una especie de anillos brillantes de austenita retenida.

Otro aspecto a señalar lo constituye en todos los regímenes analizados, el mayor espesor transformado que se presentó en la fundición laminar respecto a la nodular. Este resultado ha sido obtenido por algunos autores para velocidades inferiores a los 10 $\mathrm{mm} / \mathrm{s}{ }^{[9]}$ y puede ser atribuido al tamaño y cantidad de grafito de las fundiciones ensayadas.

Como es conocido, los nódulos de grafito presentan gran dificultad a ser disueltos totalmente. Este resultado parece estar justificado porque en las regiones que circundan el nódulo de grafito, la solución sólida de austenita presenta un contenido de carbono de $2-2,3 \%$ y puede, por tanto, tener una alta estabilidad. Otro detalle significativo está relacionado con una caída más abrupta de la dureza en profundidad de la fundición nodular, que puede estar relacionada con la disolución de la cementita, el rápido calentamiento, la razón de enfriamiento y la nueva precipitación de la fase.

\section{ENSAYOS TRIBOLÓGICOS}

Los resultados de los ensayos de desgaste para cada uno de los materiales ensayados se ofrecen en la figura 8 . 

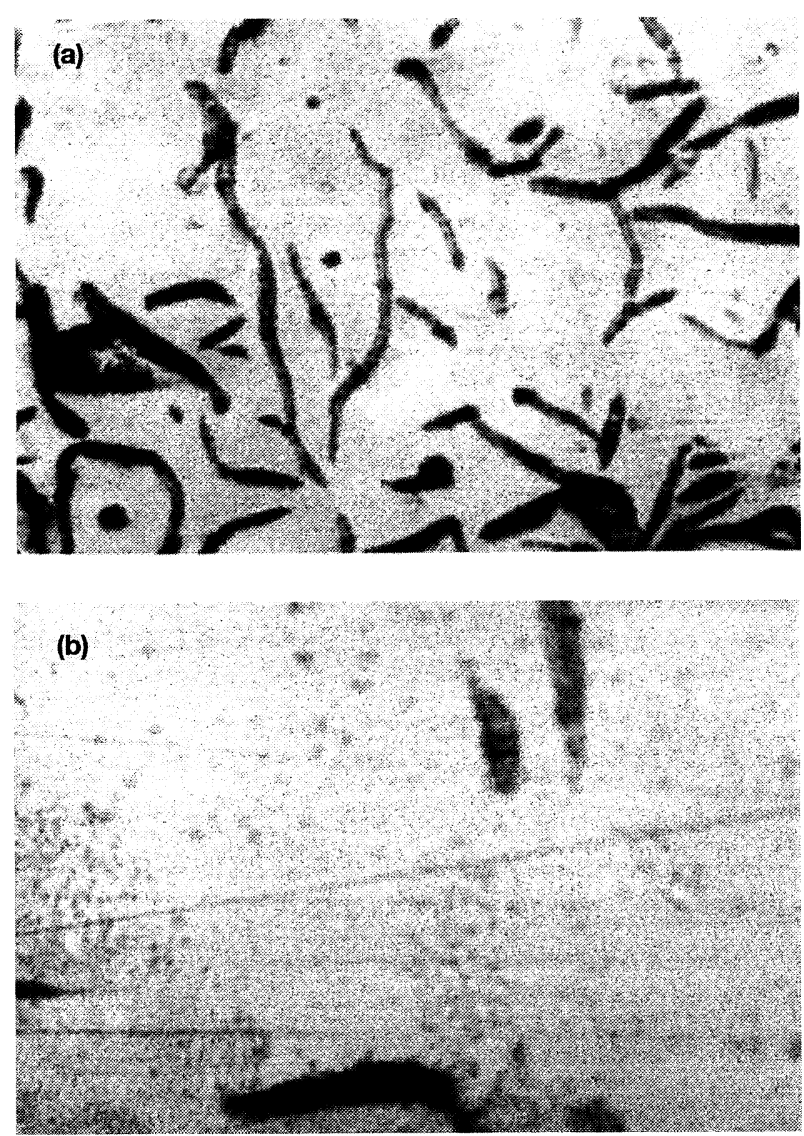

Figura 6. Microestructura de la fundición gris laminar con TSL. a) Láminas de grafito en matriz martensítica (100 X), b) Láminas de grafito parcialmente disueltas y substrato de perlita laminar (500 X).

Figure 6. Microstructure of cast iron flake with TTSL. a) Graphite flakes in a martensitic matrix (100 X), b) Graphite flakes partially dissolved and substrate of lamellar perlite (500 X).

En todos los casos se presentaron mejorías en la resistencia al desgaste de los materiales ensayados. En todos los pares tribológicos acero-acero prevaleció el tipo desgaste abrasivo, como se puede observar en la huella de desgaste de las probetas del acero AISI 1045 con tratamiento con láser (Fig. 9).

Sin embargo, en el grupo de los aceros aparece como excepción el acero AISI W112 con TTSL (pasada 3), en la que una vez desgastada la capa endurecida (alrededor de $60 \mu \mathrm{m}$ ), se incrementa el desgaste del sustrato blando, ocurriendo un intenso desgaste adhesivo y la transferencia de material del bloque al cilindro, observándose las cavidades resultantes del desprendimiento de partículas (Fig. 10). De aquí que el tratamiento láser sea una propuesta efectiva, sobre todo en pares no lubricados para favorecer la resistencia al desgaste adhesivo y abrasivo, siempre y cuando exista una correlación entre la dureza de los materiales del par.
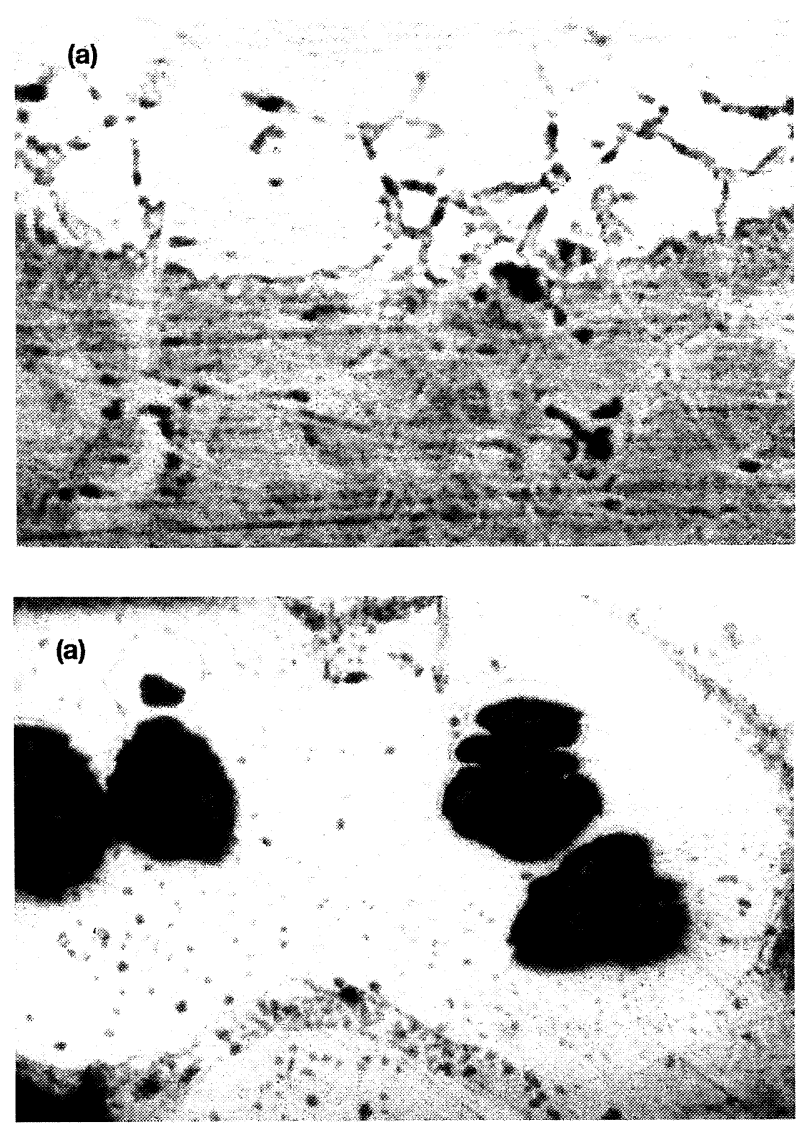

Figura 7. Microestructura de la fundición nodular después del TTSL. a) Zona afectada con martensita y grafito nodular no disuelto y substrato $(100 \mathrm{X})$, b) Nódulo de grafito rodeado de austenita retenida en una matriz martensítica $(500 \mathrm{X})$.

Figure 7. Microstructure nodular cast iron after TSL. Affected zone, martensite with nodular graphite not dissolved and substrate. Nodular graphite surrounded of retained austenite in martensitic matrix.

En el caso de las fundiciones patrones y con tratamiento láser, predominó igualmente el desgaste abrasivo y en menor medida el adhesivo, como resultado de la semicompatibilidad con los aceros entre otros aspectos por la presencia del grafito. Ambas fundiciones con TTSL presentaron cierta similitud en cuanto a su resistencia al desgaste con el acero AISI 1045 con tratamiento láser y, como era de esperar, evidenciaron un comportamiento muy superior a las fundiciones patrones.

La variación del coeficiente de fricción de las muestras ensayadas se presenta en la figura 11 .

Como puede observarse, el coeficiente de fricción, $f$, presentó un comportamiento muy similar en los aceros, con una tendencia favorable a la disminución del coeficiente de fricción respecto a los tratamientos convencionales, como se ha informado por otros autores ${ }^{[4 \mathrm{y} 8]}$. El par tribológico W112 (con 


\section{AISI 1045}

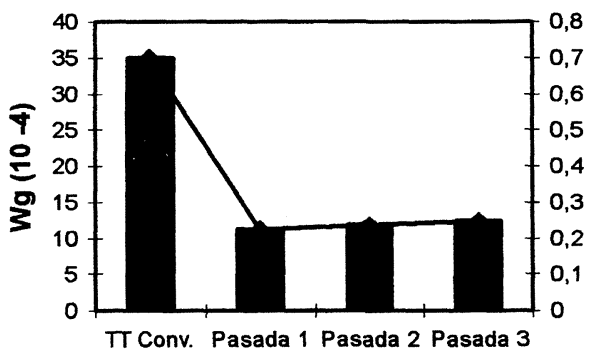

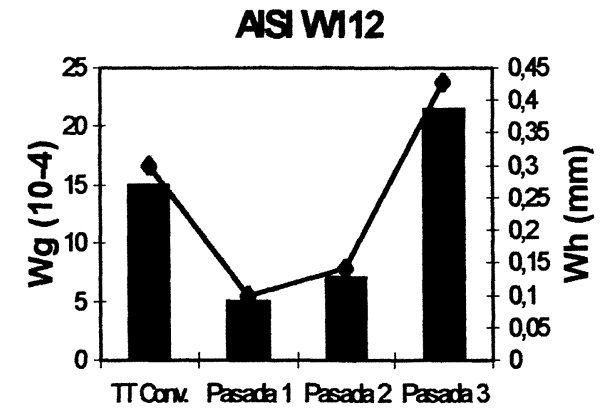

ASI W1

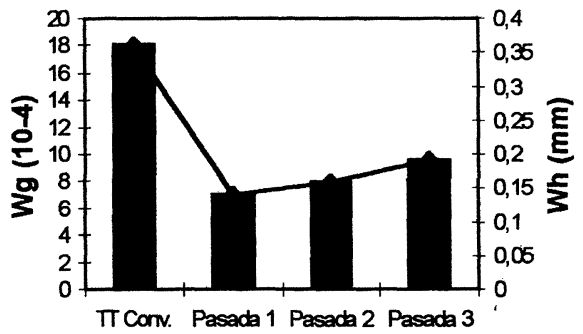

Fundición Nodular

Fundición Laminar
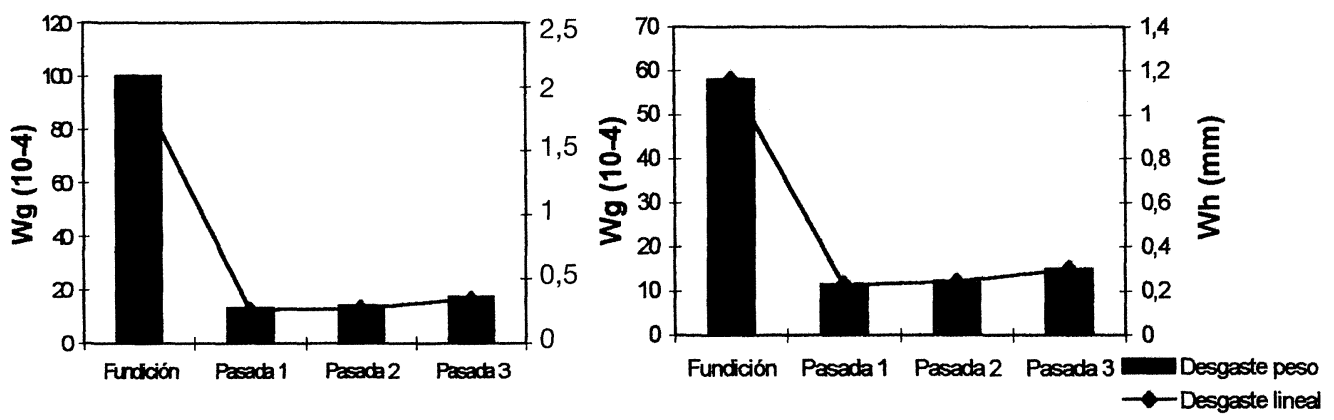

Figura 8. Desgaste gravimétrico y lineal de las muestras con tratamiento con láser y convencional.

Figure 8. Gravimetric and lineal wear for conventional and laser treatment.

TTSL)/acero 65Mn4, sin embargo, mostró, a partir de los 20 min de ensayo, un crecimiento continuo de $f$ atribuible al desgaste total de la capa endurecida y el incremento de la adhesión molecular.

Las fundiciones patrones, por su parte, presentaron los menores valores del coeficiente de fricción, debido a la acción lubricante del grafito, con los menores valores en la fundición nodular no tratada. Las fundiciones laminar y nodular con TTSL mostraron una tendencia a incrementar $f$ respecto a las fundiciones patrones, aspecto que está relacionado con la disolución parcial de láminas y nódulos de grafito.

\section{CONCLUSIONES}

- En todos los casos de los aceros tratados, la dureza en las capas superficiales osciló entre los 500 $916 \mathrm{HV}$, coincidiendo los mayores valores de dureza con los aceros de mayor contenido de carbono. Las estructuras metalográficas se caracterizaron por una martensita fina en la zona de total transformación, una más burda en la zona de transición con presencia de perlita, ferrita y carburos en diferentes grados.

- En los dos tipos de fundiciones analizadas (laminar y nodular), las durezas alcanzadas fueron 


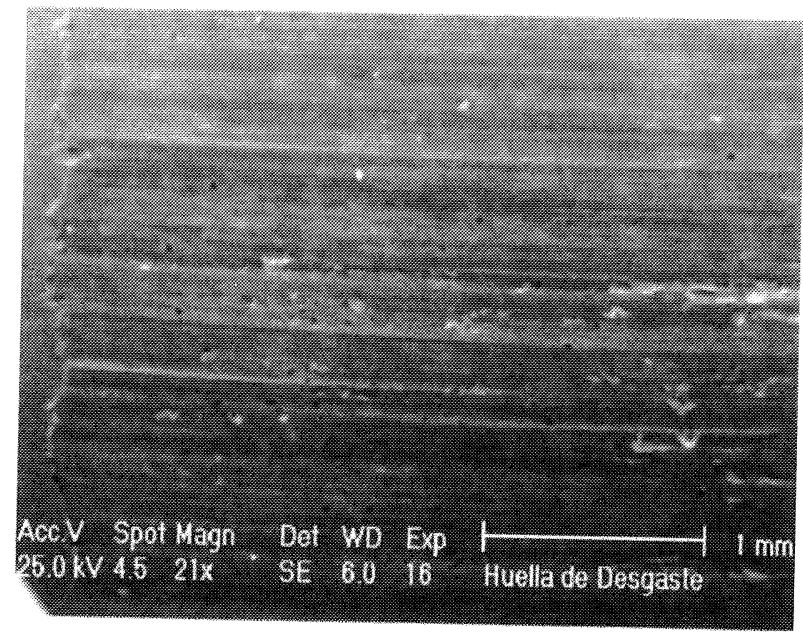

Figura 9. Desgaste abrasivo en probetas de acero AISI 1045 con tratamiento láser.

Figure 9. Abrasive wear in AISI 1045 steel with laser treatment.

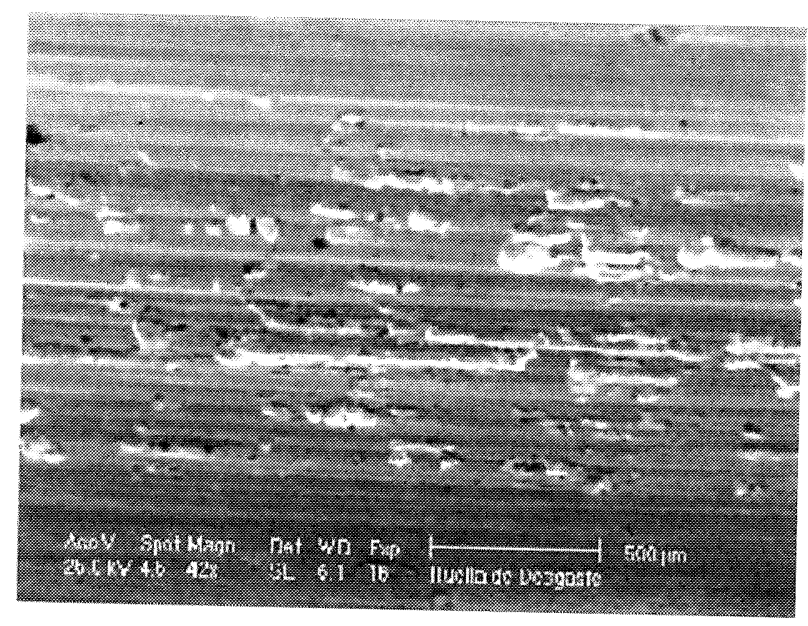

Figura 10. Desgaste adhesivo como resultado de la destrucción de la capa endurecida.

Figure 10. Adhesive wear as a result of a rapid destruction of hardening layer.

muy similares $(\approx 700 \mathrm{HV})$, y una estructura metalográfica matizada por un alto grado de disolución del grafito en la fundición laminar y la presencia del nódulo de grafito y austenita retenida en el hierro nodular en una matriz martensítica.

- Se comprobó que el aumento de la velocidad de barrido provocó una disminución del espesor de la capa endurecida.

- La resistencia al desgaste de las muestras tratadas con láser presentó un incremento significativo respecto a los tratamientos convencionales

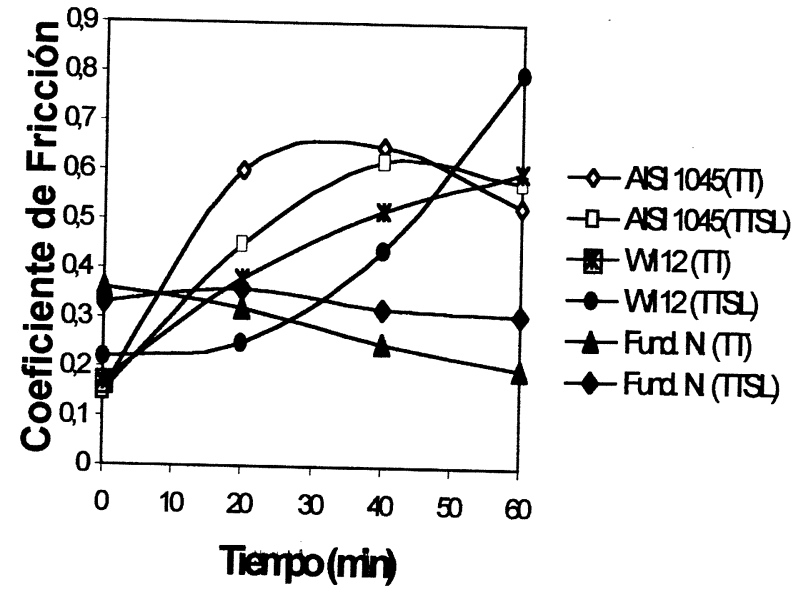

Figura 11. Comportamiento del coeficiente de fricción de las muestras ensayadas.

Figure 11. Frictional behaviour of tested samples.

y fundiciones patrones. En el caso del acero AISI' W112 (pasada 3), la insuficiente penetración del endurecimiento provocó que, una vez que ocurre la ruptura de la capa endurecida, se incrementa el desgaste del substrato blando, presentándose el desgaste adhesivo intenso y la transferencia de material del block al cilindro. En el resto de los materiales predomina el desgaste de tipo abrasivo y en menor medida el adhesivo.

- El comportamiento del coeficiente de fricción presentó una tendencia a la disminución de los valores de $f$, con la sola excepción de las fundiciones tratadas con láser en las que los más altos valores pueden estar justificados por el proceso de disolución de las láminas y nódulos de grafito. El acero AISI W112 (pasada 3) presentó, durante los ensayos, un crecimiento acelerado del coeficiente de fricción, como resultado de la destrucción de la capa endurecida y la intensificación de los procesos de adhesión molecular.

\section{REFERENCIAS}

[1] J.J. Damborenea, Segundo Curso de Ingeniería de Superficies, CSIC, España, 1998.

[2] W. STEEN, Met. Technol. (1979) 456-462.

[3] H.M. Shang, J. Mater. Processing Technol. 23 (1990).

[4] J.S. Ceballos, G. Muñiz, R. Sagaró, B. Barja y C. TORRES, Rev. Metal. Madrid 34, 2 (1998) 140-143. Edición especial de la I Conferencia Iberoamericana de Síntesis y tratamiento de materiales por láser, CSIC, Madrid, España. 
[5] V. LÓPEZ, Influence of previous structure on laser surface hardening of AISI 1045 steel, ISIJ International V-35, 1995, pp. 1394-1399.

[6] J. RuIz, Mater. Des. 17, 5-6 (1996) 267-273.

[7] B. BusHam, Handbook of Tribology: Materials, coatings and surface treatments, McGraw- Hill, Inc., 1991.
[8] H. VISSCHER, The influence of laser line hardening of carbon steel AISI 1045 on lubricated wear against steel AISI 52100, Wear of Materials Conference, Massachusetts, E.E.U.U, 1995.

[9] P. SchaAF, J. Mater. Sci. 26 (1991) 5.019-5.024. 